\title{
Interrelationships between spontaneous and low-level stimulus-frequency otoacoustic emissions in humans
}

\author{
Christopher Bergevin \\ Department of Otolaryngology/Head and Neck Surgery, \\ Columbia University, New York, NY 10032 \\ Analydia Fulcher, Susan Richmond, David Velenovsky \\ Department of Speech, Language, \& Hearing Sciences \\ University of Arizona, Tucson, AZ 85705 \\ Jungmee Lee \\ Department of Communication Sciences and Disorders \\ Northwestern University, Evanston, IL 60208
}

Version: March 5, 2012

Corresponding Author:

Christopher Bergevin

Address: 630 West 168th Street P\&S 11-452, New York, NY 10032

Email: dolemitecb@gmail.com 


\begin{abstract}
It has been proposed that OAEs be classified not on the basis of the stimuli used to evoke them, but on the mechanisms that produce them [44]. One branch of this taxonomy focuses on a coherent reflection model and explicitly describes interrelationships between spontaneous emissions (SOAEs) and stimulus-frequency emissions (SFOAEs). The present study empirically examines SOAEs and SFOAEs from individual ears within the context of model predictions, using a low stimulus level (20 dB SPL) to evoke SFOAEs. Emissions were recorded from ears of normalhearing young adults, both with and without prominent SOAE activity. When spontaneous activity was observed, SFOAEs demonstrated a localized increase about the SOAE peaks. The converse was not necessarily true though, i.e., robust SFOAEs could be measured where no SOAE peaks were observed. There was no significant difference in SFOAE phase-gradient delays between those with and without observable SOAE activity. However, delays were larger for a $20 \mathrm{~dB}$ SPL stimulus level than those previously reported for $40 \mathrm{~dB}$ SPL. The total amount of SFOAE phase accumulation occurring between adjacent SOAE peaks tended to cluster about an integral number of cycles. Overall, the present data appear congruous with predictions stemming from the coherent reflection model and support the notion that such comparisons ideally are made with emissions evoked using relatively lower stimulus levels.
\end{abstract}


List of abbreviations

- DPOAE - distortion-product otoacoustic emission

- eOAE - evoked otoacoustic emission

- $N_{\mathrm{SFOAE}}-\mathrm{SFOAE}$ phase-gradient delay expressed in stimulus periods

- $N_{\mathrm{SOAE}}-$ geometric mean frequency between adjacent SOAE peak pairs divided by their frequency separation

- $R R_{\text {stapes }}$ - product of the reflectances at the stapes and the peak of the traveling wave

- SFOAE - stimulus-frequency otoacoustic emission

- SOAE - spontaneous otoacoustic emission

- TEOAE - transient-evoked otoacoustic emission 


\section{Introduction}

Sounds evoked from the ear, known as otoacoustic emissions (OAEs), provide a noninvasive window into the mechanics of hearing [41] and are readily measurable across a wide range of species [24]. Furthermore, the ears of many normal-hearing individuals emit spontaneously (SOAEs) [22, 62]. In humans, these emissions tend to be narrow-band in nature, spectrally unique to a given ear, and relatively stable over long periods of time [9]. Additionally, SOAEs have been shown to demonstrate statistical properties consistent with self-sustained sinusoids [7, 46], suggestive as evidence for an amplification process at work in the ear. However, the study of SOAEs for evaluating cochlear status has been limited, presumably due to their relatively low incidence in normal-hearing individuals: human SOAEs occur in roughly 60-80\% of women and 25-60\% of men $[36,61,53]^{1}$. When SOAEs are present, they are typically sparsely distributed and at idiosyncratic frequencies, making them difficult to use for audiological screening purposes.

Evoked emissions (eOAEs) have demonstrated clinical value [41] and are thus more commonly measured than SOAEs. Traditionally, distortion product and transient-evoked OAEs (DPOAEs, TEOAEs) are routinely examined, despite complexities associated with their generation stemming from the multi-frequency evoking stimuli and the nonlinearity of the cochlea. For example, DPOAEs have been demonstrated to arise from at least two distinct sources in the cochlea, both of which can interfere in a complex fashion [55]. Less commonly measured are stimulus-frequency otoacoustic emissions (SFOAEs), arguably the simplest type of evoked emission, arising in response to a single stimulus tone $[23,65,34,64,48,19]$. SFOAEs have been recently demonstrated to objectively provide estimates of cochlear filter tuning bandwidths [47, 17]. However, technical difficulties associated with their measurement have limited their study ${ }^{2}$, as has controversy over

\footnotetext{
${ }^{1}$ Quantitatively, the precise incidence depends strongly upon the detection method employed (e.g., microphone noise, averaging technique) [53]. Racial differences in SOAE incidence have also been noted [61], as well as lateralization (SOAEs are more common in the right ear rather than the left) $[8,26]$ and variations with heartbeat [31].

${ }^{2}$ SFOAEs arise at the same frequency as the stimulus being used to evoke them, thereby making them difficult to extract. Typically a suppression paradigm is employed (see Methods), but such a method can be more sensitive to nonlinearities associated with the measuring earphone(s) and mic. However, there are numerous other methods by which SFOAEs can be extracted, all leading to the same result when low stimulus levels are employed [18].
} 
interpretation of SFOAE generation [e.g., [49]].

Previous theoretical studies have suggested that low-level SFOAEs arise primarily from a linear process of coherent reflection $[63,56]$. These reflections are hypothesized to arise due to random perturbations along the cochlea, or an inherent roughness of the cochlear partition [32]. A taxonomy classifying emissions posits that SOAEs and SFOAEs arise fundamentally from the same mechanism [44], SOAEs being a special self-sustained case where the cochlea behaves in a manner analogous to a laser-cavity [46]. While such a hypothesis was tested empirically and found to hold well [46], a stimulus (probe) level of $L_{\mathrm{p}}=40 \mathrm{~dB}$ SPL was used to evoke the SFOAEs. As motivated by [38], [46] argued for an "intensity correction" to the data, a step that should be unnecessary if a lower probe level is used. Another motivation for using lower stimulus levels stems from the nonlinear nature of the cochlea. In light of empirical observations that mammalian basilar membrane growth functions are linear for low stimulus levels, but become compressive above $\sim 20-30 \mathrm{~dB}$ SPL [42], a moderate stimulus level of $L_{\mathrm{p}}=40 \mathrm{~dB}$ SPL may introduce nonlinear effects that could confound relating data to model predictions. In fact, human SFOAE phase-gradient delays have been shown to increase further with decreasing stimulus level $[63,43,2]$, though delays have not been examined in detail for stimulus levels below $40 \mathrm{~dB}$ SPL. One additional consideration is that comparisons between SOAEs and SFOAEs made by [46] were examined in grouped data pooled across subjects, not data from individual ears. Such comparisons in individuals would provide additional ways to test model predictions, by quantifying features such as how SFOAE phase changes about and between adjacent SOAE peaks.

The goal of the present study is to examine several questions within the context of SOAEs and SFOAEs interrelationships, as motivated by the coherent reflection [63] and global standing wave models [46] for emission generation. Specifically:

- What correlations exist between SOAEs and SFOAEs in individual ears? For example: Do SOAEs occur where SFOAE magnitudes are largest? How much SFOAE phase accumulation occurs between SOAE peaks? 
- How do SFOAEs compare between subjects with and without strong SOAE activity?

- How do the properties of SFOAEs evoked with lower stimulus levels (20 dB SPL) compare to results of other studies using similar methods, but higher stimulus levels (e.g., $40 \mathrm{~dB}$ SPL)?

Regardless of any specific model, addressing these empirically-based questions will shine further light upon the biophysical mechanisms at work in the ear responsible for OAE generation.

\section{Methods}

\subsection{Measurement System}

All measurements reported here were obtained using similar methods as those reported previously $[44,3]$. A desktop computer housed a 24-bit soundcard (Lynx TWO-A, Lynx Studio Technology), whose synchronous root mean square (RMS) input/output was controlled using a custom data-acquisition system. A sample rate of $44.1 \mathrm{kHz}$ was used to transduce signals to/from a probe containing an Etymotic ER-10A microphone and two ER2-A earphones ${ }^{3}$. The microphone was calibrated using a pistonphone (Brüel and Kjær, type 4230). For OAE experiments, mic responses were amplified by $40 \mathrm{~dB}$ and high-pass filtered with a cut-off frequency of $0.41 \mathrm{kHz}$ to minimize the effects of noise. The OAE probe was coupled to the external ear by means of a rubber tip. This ensured a tight (closed) acoustic coupling and minimized low-frequency losses $(<1 \mathrm{kHz})$. The probe earphones were calibrated in-situ by presenting flat-spectrum, random-phase noise. Calibrations were verified repeatedly throughout the experiment. All stimulus frequencies were quantized such that an integral number of cycles were contained within the sampling window. All testing was conducted in a sound-attenuating booth.

\footnotetext{
${ }^{3}$ A small subset of data, not included in the final analysis, was also obtained using an ER-10C. Similar results for a given ear were obtained from both probe assemblies.
} 


\subsection{Measurement and Analysis Paradigm}

Subjects were typically seated quietly in the booth for an initial period of at least 10-15 minutes before testing [60]. SOAE data were typically collected at the start of an experiment, though in several cases they were collected both before and after SFOAE data collection. No differences were generally seen in those cases. A total of 60 waveforms (32768 sample window, $\mathrm{SR}=44.1 \mathrm{kHz}$ ) were acquired and the FFT magnitudes spectrally averaged, either with or without a suppressor tone present. The presence of SOAE peaks was typically confirmed by presenting a nearby tone (40 dB SPL) and observing suppression.

For SFOAEs, the range of stimulus frequencies $\left(f_{\mathrm{p}}\right)$ employed was typically $0.5-5 \mathrm{kHz}$, depending upon the location of SOAE activity. The frequency step of $\sim 15 \mathrm{~Hz}$ was chosen as a compromise between obtaining SFOAE data in a reasonable amount of time and trying to avoid ambiguities in phase unwrapping (particularly about SOAE peaks, where SFOAE phase can rotate rapidly). The stimulus and emission frequency are one and the same for SFOAEs. A two-tone suppression paradigm was employed to extract the SFOAE [44]. A stimulus level of $L_{\mathrm{p}}=20 \mathrm{~dB}$ SPL was used. The suppressor parameters were: $f_{\mathrm{s}}=f_{\mathrm{p}}+40 \mathrm{~Hz}, L_{\mathrm{s}}=L_{\mathrm{p}}+15 \mathrm{~dB}$ (where $f_{\mathrm{s}}$ and $L_{\mathrm{S}}$ are the suppressor frequency and level, respectively). It is noted that while a previous SFOAE study [19] examined low stimulus levels ( $L_{\mathrm{p}} \leq 40 \mathrm{~dB}$ SPL), in contrast to the present study they used a fixed suppressor level $\left(L_{\mathrm{s}}=55 \mathrm{~dB}\right.$ SPL). The reasoning for using a fixed $L_{\mathrm{s}}-L_{\mathrm{p}}$ (and thereby $L_{\mathrm{s}}=35 \mathrm{~dB}$ SPL) here was that, given the nonlinearity of the cochlea that manifests at moderate to high stimulus levels, by keeping all presented stimuli as relatively low as possible unanticipated nonlinear effects would be reduced.

A total of 35 waveforms (8192 sample window) were averaged, excluding any flagged by an artifact-rejection paradigm [44]. A period of $\sim 20 \mathrm{~ms}$ was allowed before the start of the sample window, allowing for the associated response to reach steady-state. Propagation of error in the suppression paradigm was computed across the averages to compute uncertainty via the standard error of the mean [2]. Frequency step-size during sweeps was small enough to avoid ambiguity 
during the phase unwrapping. Delays associated with the measurement system were determined and subtracted out. The noise floor was defined as the average sound-pressure level centered about (but excluding) the frequency of interest. It was quantified via averaging the magnitudes of the \pm 3 bins about the probe in the fast Fourier transform (FFT) of the response.

The phase-gradient delay, expressed either in time ( $\left.\tau_{\mathrm{OAE}}\right)$ or number of stimulus periods $\left(N_{\mathrm{SFOAE}}\right)$, is obtained from the derivative of the phase function with respect to probe frequency $\left(f_{\mathrm{p}}\right)$. It is given by

$$
N_{\mathrm{SFOAE}}=f_{\mathrm{p}} \tau_{\mathrm{OAE}}=-\frac{f_{\mathrm{p}}}{2 \pi} \frac{\partial \phi_{\mathrm{OAE}}}{\partial f_{\mathrm{p}}}
$$

where $\phi_{\mathrm{OAE}}$ is the emission phase (in radians) and $f_{\mathrm{p}}$ is in hertz. Delays (i.e., $\tau_{\mathrm{OAE}}$ ) were computed from individual (unwrapped) phase responses using centered differences [45]. Only unique data from each ear were included (i.e., no repeats). Phase-gradient delays were corrected for the acoustic delay due to the distance in the canal between the probe and tympanic membrane (assumed 2 $\mathrm{cm}$ for all subjects). Delay trends were computed via a locally-weighted regression (loess). To further reduce the effects of outliers at the end points, only $N_{\mathrm{SFOAE}}$ values whose corresponding magnitude (as well as the magnitude of its neighbors) was at least $10 \mathrm{~dB}$ above the noise floor were included in the fits. Uncertainty in the loess trends was computed via bootstrapping: By randomly re-sampling the data (using the same total number of points) and recomputing the trend, the corresponding standard deviation across fits could be determined. Such computations provided the corresponding 95\% confidence intervals. Additional details regarding the computation of the loess fits can be found elsewhere [45, 3].

Analysis required estimation of SOAE peak frequencies and the SFOAE phase at those frequencies. SOAEs were determined to be present when there was a peak whose shape was consistent with previous reports [e.g., Fig.6 in [46]] and was at least $3 \mathrm{~dB}$ above the noise floor ${ }^{4}$. Second, estimating the SFOAE phase at the SOAE frequencies involved a simple linear interpolation (see

\footnotetext{
${ }^{4}$ Unfortunately, these conservative criteria likely excluded some smaller, more broadband peaks that may have been more readily (and justifiably) detectable using different extraction methods [53, 58].
} 
Fig.4A). These values were then used to compute the SFOAE phase difference between adjacent SOAE frequencies ( $\Delta \phi$ in Fig.4).

\subsection{Subjects}

All subjects were normal-hearing adults (between the ages of 19 and 39, mean age 23 years old) and passed pure-tone hearing screening $(0.5-4 \mathrm{kHz}$ at $15 \mathrm{~dB} \mathrm{HL})$ and had normal tympanograms (except for one particular subject; see Results). Data from a total of 16 different subjects (22 unique ears) are included here. Of these, eight subjects exhibited robust SOAE activity (3 or more peaks meeting the above criteria) in at least one ear, two mild activity ( 1 or 2 peaks), and six had no measurable activity at all in either ear. However, these numbers are somewhat biased in that early in the study, several subjects were excluded from SFOAE measurements due to a lack of SOAE activity. All subjects were female except for two. As noted above, subjects sat quietly in the booth long enough to allow any potential adaptation effects to subside ${ }^{5}$. Though SOAEs can be affected by aspirin consumption $[35,29,30]$, this was not controlled for int he present study.

\section{Results}

Figure 1 shows a comparison of SOAEs and SFOAEs for several different representative individuals. Panels A and B indicate two subjects that had both robust SOAE and SFOAE activity. Panel C shows an example of a subject who demonstrated robust SFOAEs, but little SOAE activity apparent. While this type of behavior was observed in about $\sim 35 \%$ of all the ears examined, several subjects were excluded from SFOAE data acquisition due to lack of SOAE activity. Thus, the true distribution can not be inferred due to such a bias. Note that several small, wideband peaks may be just discernible in the SOAE spectrum of panel C (e.g., 1.96, 3.2, and $3.7 \mathrm{kHz}$ ) [see [53]]. Conversely, panel D shows data from the only subject (39 year old female) who bilaterally had

\footnotetext{
${ }^{5}$ We observed early in the study that SOAEs in subjects, who are known to posses spontaneous activity, can be reduced or altogether absent without such a 'settling' period. This effect appears to be associated with the acoustic environment they were in immediately before coming to the lab (i.e., noisy environments or exposure to loud sounds caused greater reduction), and presumably is due to some sort of slow adaptation effect [60].
} 

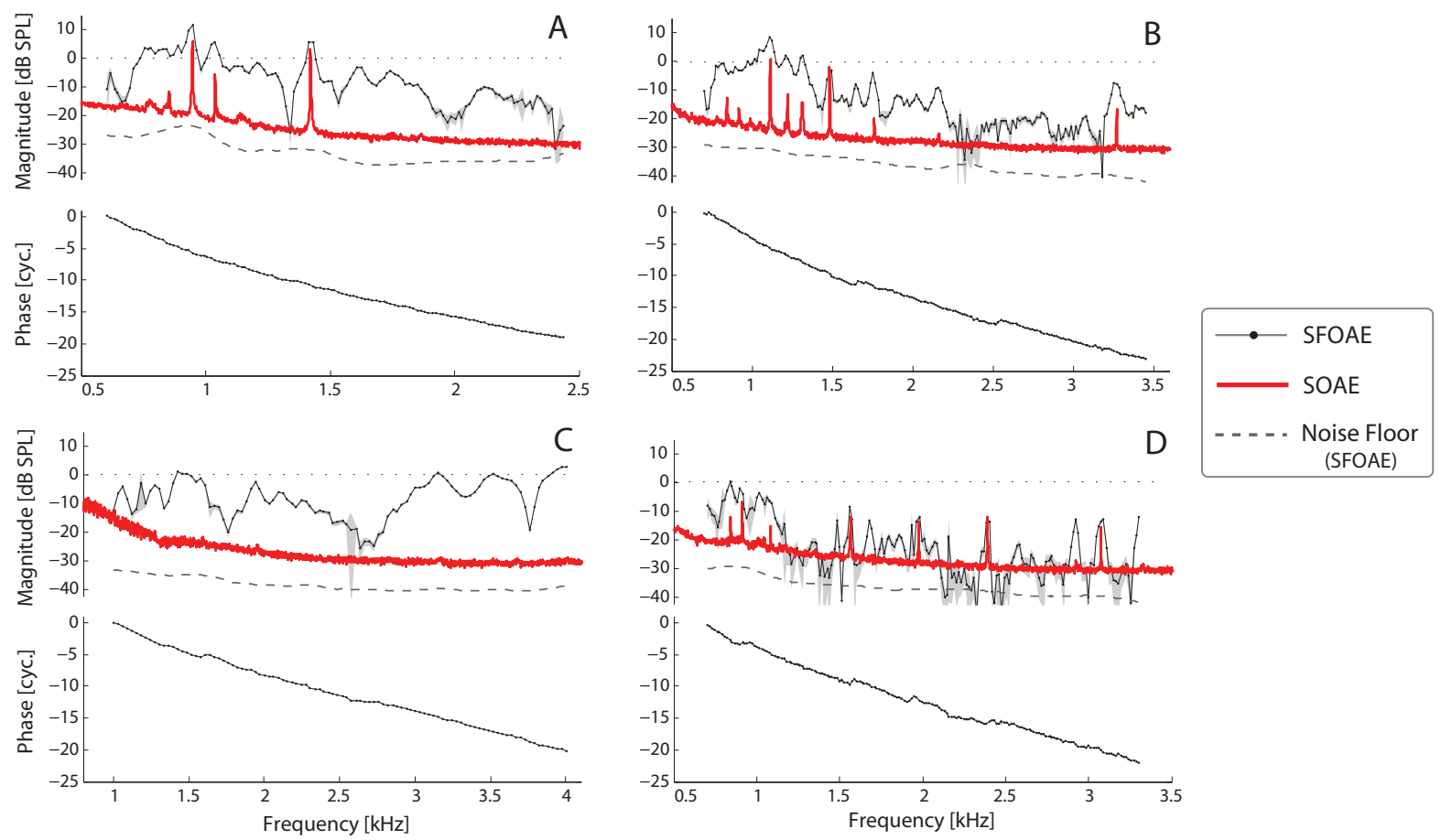

Figure 1: Comparison of SOAEs and SFOAEs (evoked via a $20 \mathrm{~dB}$ SPL stimulus tone) for four normal hearing adults. Dashed lines indicate the noise floor for the (time-averaged) SFOAE recordings. Uncertainties are shown via the grey shading, which indicate the standard error of the mean across the number of waveforms averaged for a given frequency. Panels A and B show subjects who exhibited both SOAE and SFOAE activity. Panel C indicates a subject who had little SOAE activity, but robust SFOAEs. Conversely, panel D shows the one subject in the study who had strong SOAE activity, yet SFOAEs were relatively small. The subject in panel D also had an abnormal tympanogram, despite normal audiometric thresholds (see text). Note the different abscissa scales across panels. 

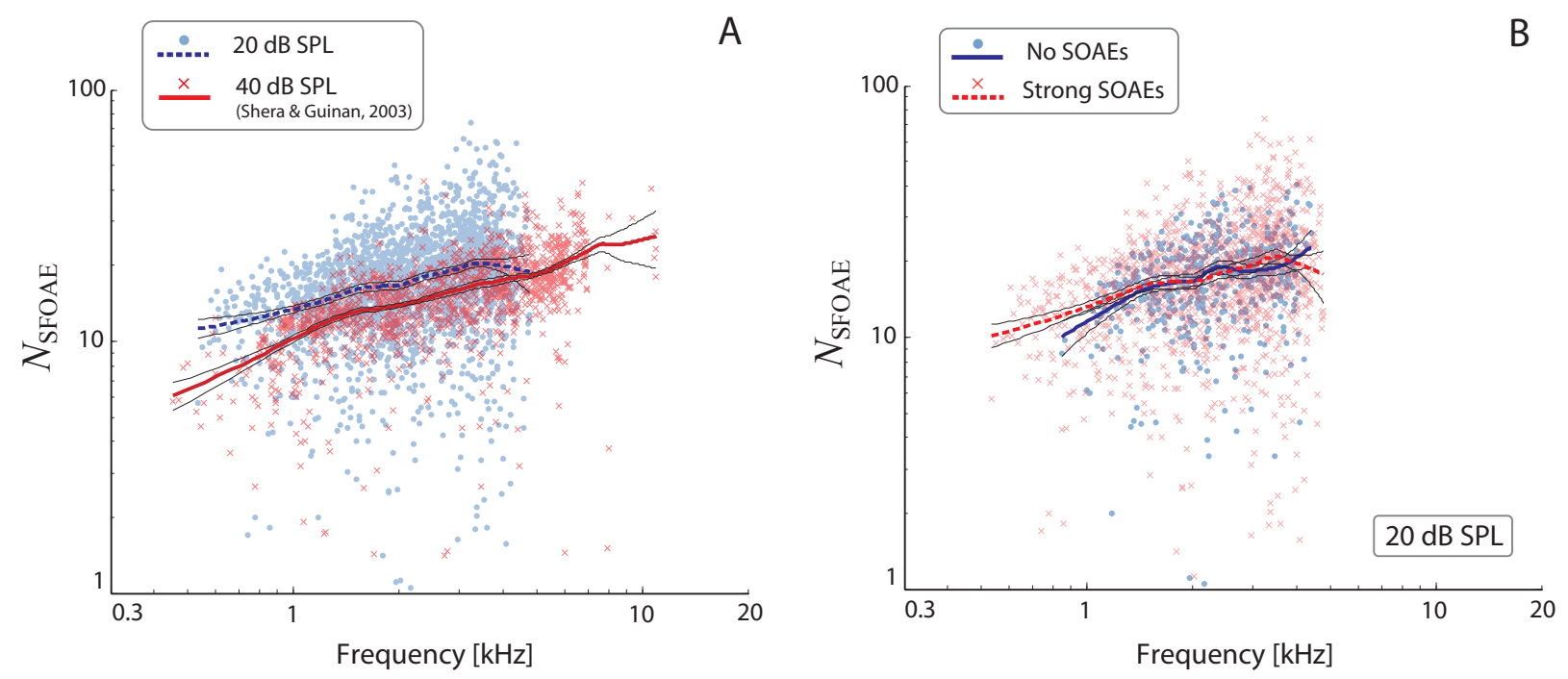

Figure 2: Comparison of SFOAE phase-gradient delays $\left(N_{\mathrm{SFOAE}}\right)$. In both panels, thick curves show loess trend lines along with $95 \%$ confidence intervals (see Methods), where only points whose magnitudes was at least $10 \mathrm{~dB}$ above the noise floor are included. A - Comparison of present data collected at $L_{\mathrm{p}}=20 \mathrm{~dB}$ SPL ( $\mathrm{m}=16$ different subjects, $\mathrm{n}=22$ unique ears) versus those collected at $L_{\mathrm{p}}=40 \mathrm{~dB}$ SPL from a previous study [45] that used similar methods. B - Comparison of subjects within the present study ( $L_{\mathrm{p}}=20 \mathrm{~dB}$ SPL), differentiated between those showing robust SOAE activity (at least three peaks, $10 \mathrm{~dB}$ or more above the noise floor; $m=8, n=11$ ) versus those who showed none $(m=6, n=8)$.

robust SOAE activity, but relatively small SFOAEs. While this subject had normal audiometric thresholds, she had a history of chronic otitis media with effusion and multiple tympanic membrane ruptures during childhood. This subject was the only one in the study with an abnormal tympanogram, which indicated a hypermobile tympanic membrane. In all subjects, SFOAEs were readily detectable and the SFOAE phase exhibited many cycles of accumulation as the stimulus tone was swept (Fig.1), indicative of delays on the order of several milliseconds long. Measurements from an individual were stable within a recording session, at least beyond the initial settling time as noted in the Methods. While longer term stability was not examined in detail, emissions appeared generally reproducible across sessions in several individuals where such recordings were made.

The SFOAE phase-gradient delays, expressed as dimensionless $N_{\mathrm{SFOAE}}[47,6]$, are shown in Figure 2. Two different comparisons are made. In panel A, the present data $\left(L_{\mathrm{p}}=20 \mathrm{~dB} \mathrm{SPL}\right)$ are plotted along with those from a previous study [45] that used similar methods, but a higher 
stimulus level $\left(L_{\mathrm{p}}=40 \mathrm{~dB} \mathrm{SPL}\right)^{6}$. The delays are systematically longer in the case of lower stimulus levels, consistent with previous reports $[56,43,2]$. Comparing the trends from $1-4 \mathrm{kHz}$, the $L_{\mathrm{p}}=20 \mathrm{~dB}$ SPL delays are larger by a factor of 1.17-1.3 compared to the $L_{\mathrm{p}}=40 \mathrm{~dB}$ SPL case, this ratio not exhibiting a strong dependence upon frequency. Note the larger variance apparent in the low-level data of Fig.2A, which may reflect the fact that the magnitudes are closer to the noise floor and dip down into it (thereby causing random discontinuities into the unwrapped phase). In panel $\mathrm{B}$, the present data are broken up into two groups: individuals with robust SOAE activity (at least three peaks, each $10 \mathrm{~dB}$ or more above the noise floor) and those with no apparent SOAEs. There was no systematic offset in the noise floor across the groups, indicating that the lack of SOAEs in one group was not simply due to an increased noise floor. Statistically the two groups are indistinguishable (as indicated by the overlap in the $95 \%$ confidence intervals), thus indicating that $N_{\mathrm{SFOAE}}$ values are similar across both groups. A slightly larger variance apparent for the strong SOAE group may reflect contributions of sudden phase jumps that sometimes occur between peaks in an individual response.

Figure 3 indicates the distribution of $N_{\mathrm{SOAE}}$ values from the present data set (70 total pairs). These values were quantified by determining the geometric mean frequency between adjacent peak pairs and dividing by the frequency separation between them [46]. Note that [46] used SOAE peak spacing data from previous studies $[53,8]$ comprising a total of 556 pairs. The present data differ from the distribution reported in [46] in that the peak is shifted towards smaller values, centered about $N_{\mathrm{SOAE}} \cong 10$. Furthermore, the present distribution shows a greater proportion of observations with smaller $N_{\mathrm{SOAE}}$ values. While the present data pool of SOAE peak pairs sampled is smaller than that of [46], the source of this discrepancy between the two studies likely stems from the different methodologies used to determine the presence SOAE peaks. The value

\footnotetext{
${ }^{6}$ Previous measurements in humans made with the same equipment/stimulus paradigms as used here, but at $L_{\mathrm{p}}=$

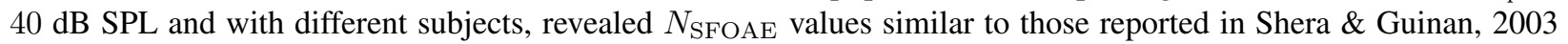
[2]. Thus, the offset shown in Fig.2A appears to be directly attributable to differences in stimulus level, not systematic differences in measurement technique or subject pools employed across studies.
} 


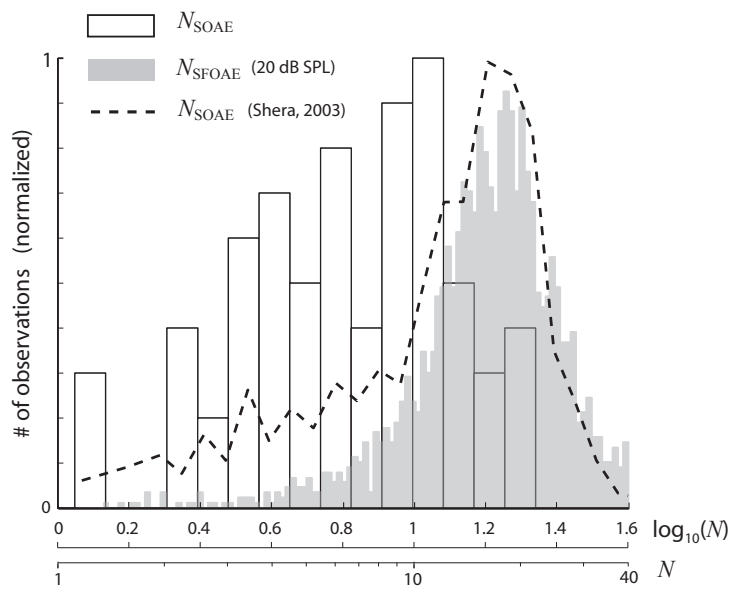

Figure 3: Distribution of $N$ values (see text). Note the normalized vertical axis. From the present study, $N_{\mathrm{SOAE}}$ was computed from a total of 70 peak pairs (histogram normalized by a factor of 1/10). The $N_{\mathrm{SFOAE}}$ values $\left(L_{\mathrm{p}}=20 \mathrm{~dB}\right.$ SPL, all subjects included) pools approximately 2000 data points (normalization factor $-1 / 80 ; N_{\mathrm{SFOAE}}<1$ excluded for clarity). Also included is the $N_{\mathrm{SOAE}}$ distribution from [46] (normalization factor $-1 / 70$; dashed line).

of $N_{\mathrm{SOAE}}$ depends critically upon the spacing between the two SOAE peaks within a pair. While $N_{\text {SOAE }}$ was only quantified for 'adjacent' pairs that met the criteria (see Methods), there could be additional peaks close to the noise floor that were not included in the present analysis [see [53]]. For example, in Fig.1B our analysis only counted seven SOAE peaks below $2 \mathrm{kHz}$, whereas visual inspection may suggest a number closer to 10 or 11 . Such smaller peak spacings would lead to higher $N_{\mathrm{SOAE}}$ values. Also shown in Fig.3 is the distribution of $N_{\mathrm{SFOAE}}$ values $\left(L_{\mathrm{p}}=20 \mathrm{~dB}\right.$ SPL), which agrees excellently with the $N_{\mathrm{SOAE}}$ distribution from [46] about the peak. Note that this correlation does not require the "intensity correction" as was used in [46] (see their Fig.4).

Figure 4 shows the direct estimation of the amount of SFOAE phase accumulation occurring between adjacent SOAE peaks $(\equiv \Delta \phi)$. Panel A indicates the general interpolation method employed to estimate the SFOAE phase for the corresponding SOAE peaks (indicated by the x symbols), since the two frequencies did not necessarily line up. As shown in panel $\mathrm{B}, \Delta \phi$ tends to cluster about an integral number of cycles (vertical dashed lines), indicating integer numbers of SFOAE phase accumulation between adjacent SOAE peaks. Panel $\mathrm{C}$ shows the same data, but modulo one cycle. The distribution has a peak about zero, but due to the relatively low number of samples (55 total) the result is not significant relative to a flat distribution (Kolmogorov-Smirnov 

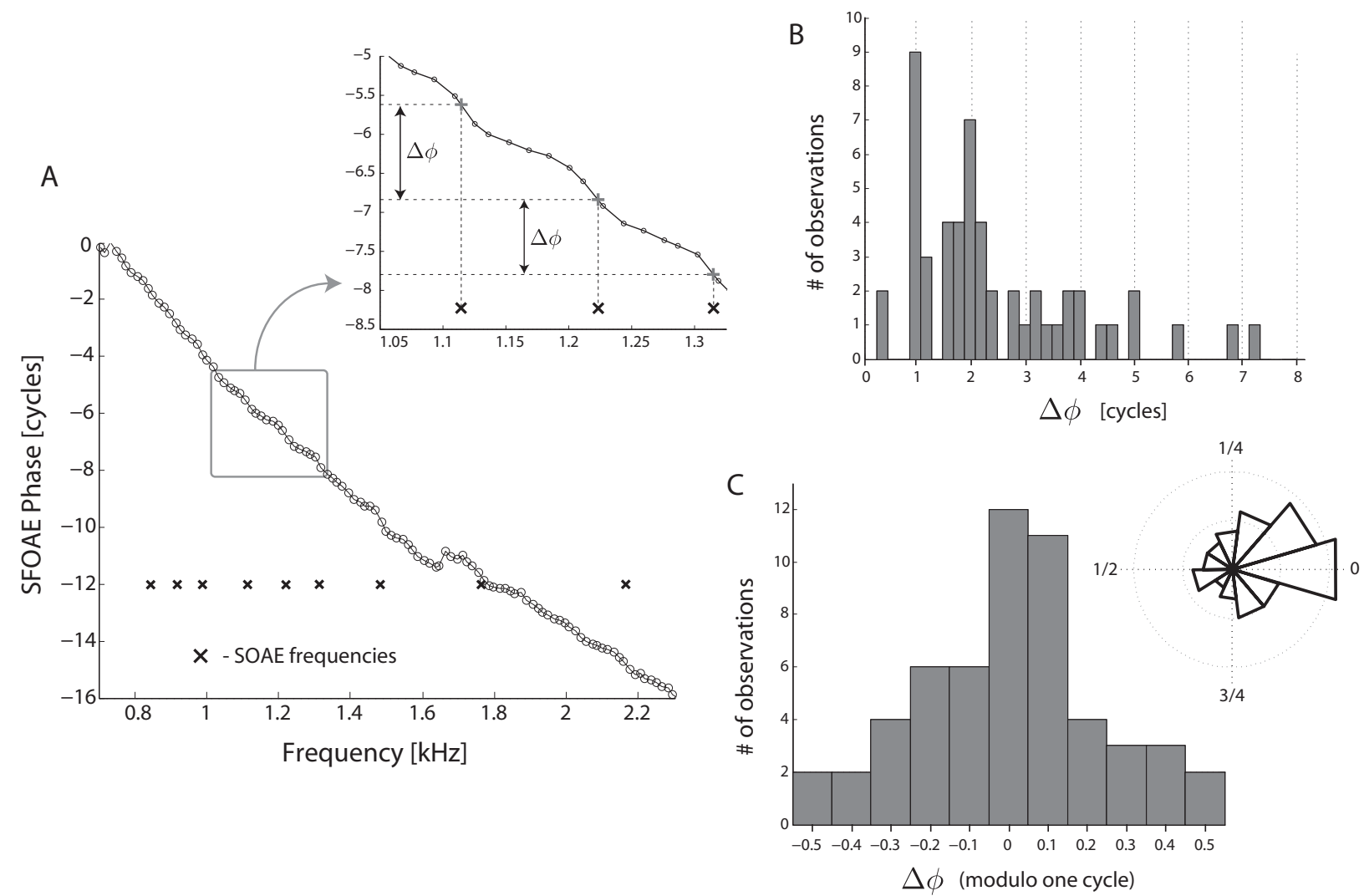

Figure 4: Distribution of SFOAE phase accumulation between adjacent SOAE peaks. A - SFOAE phase data from an individual subject (error bars excluded for clarity), to demonstrate the general method. $x$-marks indicate frequencies at which SOAE peaks were measured. Inset zooms in to show how phase values at those frequencies were interpolated (grey +-marks) so to estimate the amount of SFOAE phase accumulation that occurred between peaks $(\Delta \phi)$. B Combined data showing $\Delta \phi$ across 10 different subjects for 14 different ears. Only values up to 8 cycles are shown for clarity (higher values were observed up to 15 cycles). C - Same data as shown in panel B, but modulo one cycle. Inset shows same data as a polar distribution. 
test with a criterion probability of 0.05$)$.

\section{Discussion}

\subsection{Testing theoretical predictions}

Previous studies have suggested that SOAEs and SFOAEs are generated by the same underlying mechanism in the cochlea $[65,40,34,64,54,63,44]$, or at the least, SOAEs contribute to evoked emissions [e.g., [26]]. More recently, [46] used the coherent reflection model [63] to make (and test) several specific theoretical predictions about the interrelationships between the two emission types. That study proposed a model where self-sustained global standing waves are set up in the cochlea between the peak of the traveling wave (that has reflectance $R$ ) and the stapes (seen looking outwards from the cochlea, with reflectance $R_{\text {stapes }}$ ). While [46] indicated compelling correlations, that study did not examine SOAE/SFOAE interrelationships from individual ears and also argued that it may be preferable to test the model using SFOAE data obtained using relatively lower stimulus levels so to avoid the need for an "intensity correction". A lower stimulus level also has the benefit that the region of OAE generation is presumably more spatially confined. Such would reduce the potential for source mixing and other nonlinear effects $[65,10,16,18]$ that might confound interpretation.

As shown in Fig.1, when present, SOAE peaks always correlated to locally increased SFOAE activity. However, the converse is not always true: robust SFOAE activity can be present even in the absence of any observable SOAEs (e.g., Fig.1C). Furthermore, the SOAE amplitude does not readily correlate to that of the SFOAEs (e.g., in Fig.1B, SFOAE magnitudes are similarlysized about both small and large SOAE peaks). These observations appear generally consistent with previous empirical results $[65,40,34,48]$ as well as model considerations that suggests more specific requirements need to be met for SOAEs as opposed to SFOAEs [55, 46, 15]. While these data appear to support the global standing wave model [46], they may also be consistent with other classes of models for SOAE generation such as van der Pol oscillator frameworks that consider 
multiple generators [e.g., [37, 59]].

Spacing between SOAE peaks (Fig.3) has been demonstrated to reveal important properties of underlying cochlear mechanics and relate directly to SFOAE phase-gradient delays $[53,63,46]$. Theoretical considerations [46] predict that $N_{\mathrm{SOAE}}$ and $N_{\mathrm{SFOAE}}$ should be roughly proportional. The larger $N_{\mathrm{SFOAE}}$ values (Fig.2A) reported here for a lower stimulus level appear to narrow the gap between $N_{\mathrm{SOAE}}$ and $N_{\mathrm{SFOAE}}$ apparent in the previous report [46], which required/argued for an "intensity correction". Note the agreement here (Fig.3) that manifests without requiring such a correction. Not only does the $20 \mathrm{~dB}$ SPL probe level agree well with what was predicted by [46], but also supports that proposed correction method that was based off of previous observations made by [38]. It should also be emphasized that increased SOAE activity does not appear to readily affect $N_{\text {SFOAE }}$ values (Fig.2B). This suggests that SOAE entrainment does not bias the delays (e.g., via ringing after stimulus offset, as apparent in TEOAEs), at least in the pooled data shown here.

Another measure relevant to model predictions is shown in Fig.4, the estimation of SFOAE phase accumulation between adjacent SOAE peaks $(\Delta \phi)$. In general, $\Delta \phi$ tends to be clustered at integer numbers, indicating an integral number of cycles of SFOAE phase accumulation between SOAE peaks (Fig.4B,C). This clustering appears reminiscent of that observed in the ratios of adjacent SOAE peaks [Fig.7 of [53]]. Note that the global standing wave model [46] does not require the SFOAE phase to cluster this way, given how $\Delta \phi$ has been defined. It is the product of the two reflectances $\left(R R_{\text {stapes }}\right)$ that should exhibit this property.

Several considerations could help further strengthen conclusions drawn from $\Delta \phi$. Variations of the estimated SFOAE phase about the SOAE peak may be expected, given that SFOAE properties (both magnitude and phase) can change rapidly in the vicinity of strong spontaneous activity. Thus, even with the fine frequency resolution used here for the SFOAE measurements, the interpolation used to estimate the SFOAE phase at the SOAE frequency will likely introduce some degree of error. Consideration of the SFOAE noise floor and uncertainty could also help provide better estimates of $N_{\text {SFOAE }}$ [Shera and Bergevin; in preparation]. Lastly, another source of uncertainty 
may be the SOAE frequencies themselves. Two potential ways to better objectively estimate such would be to use an improved criteria for peak detection [53] and the use of a fitting function [e.g., a Lorentizian curve; $[53,58]]$ to the spectrum about the SOAE peak for more accurate determination of the emission frequency.

Related to this last point is the difference in $N_{\mathrm{SOAE}}$ distributions in Fig.3. Data on SOAE spacing from previous studies [primarily [53]] included smaller/wideband peaks, whose distribution closely matched that of the SFOAE phase-gradient delays (Fig.3). Inclusion of such small peaks thus appears crucial for the $N_{\mathrm{SOAE}}$ and $N_{\mathrm{SFOAE}}$ distributions to match. Consideration only of the tall/narowband SOAE peaks alone (as done in the present study) is insufficient. This observation indicates that it is important to carefully consider signal processing strategies for the identification of SOAE activity.

\subsection{Implications for larger delays at lower stimulus levels}

SFOAE phase-gradient delays have empirically been shown to increase with decreasing stimulus level [e.g., [63, 16, 43, 51, 2, 12]], a feature also observed using different methodologies [20] and explored theoretically $[56,50,12]$. This observation appears congruous with auditory nerve fiber tuning curves and basilar membrane gain functions appearing sharper when measured using lower threshold criteria. Many of these studies however employed stimulus levels of $L_{\mathrm{p}}=40 \mathrm{~dB}$ SPL and higher. Arguments made in the previous section, with regard to comparisons with SOAEs, suggest this may be too high of a level to relate to threshold responses. Several studies [19, 2, 12] have reported SFOAEs evoked using levels below $40 \mathrm{~dB}$ SPL, but questions still remain with regard to relating SFOAE phase-gradient delays to cochlear tuning and the role of stimulus level ${ }^{7}$. As shown in Fig.2A, the present data demonstrate that SFOAE phase-gradient delays $\left(N_{\mathrm{SFOAE}}\right)$ further increase when the stimulus level is lowered to $20 \mathrm{~dB} \mathrm{SPL}$ (for $L_{\mathrm{s}}=L_{\mathrm{p}}+15 \mathrm{~dB}$ ). This

\footnotetext{
${ }^{7}$ For example, [19] reported SFOAE 'transfer functions' for stimulus levels going down as far as $L_{\mathrm{p}}=10 \mathrm{~dB}$ SPL, but whose phase curves did not exhibit a level-dependent effect in their slopes. They used a fixed $L_{\mathrm{S}}=55 \mathrm{~dB}$ SPL (regardless of $L_{\mathrm{p}}$ ), which may account for discrepancies with the present study. Further study is needed to explore this observation.
} 
result suggests that delays from $L_{\mathrm{p}}=40 \mathrm{~dB}$ SPL studies may underestimate tuning bandwidths, a concern raised by others previously $[46,1]$. Additionally, studies that investigate differences in tuning estimates obtained via SFOAE phase-gradient delays versus suppression tuning curves [e.g., $[20,28,1]]$ may need to also consider probe levels lower than $40 \mathrm{~dB}$ SPL, or some sort of unmixing to reduce contributions from possible nonlinear distortion sources $[16,18]$.

When comparing SFOAEs across species [e.g., [6, 3, 17]], there may be systematic offsets as to what is the appropriate level for a given species. Or put another way, there may be differences across species where and how the low-level basilar membrane responses transitions from linear to compressive [e.g., 20-30 dB SPL for chinchilla [42]]. While the larger human delays reported here may suggest an even larger gap between humans and rhesus monkeys [17], such a conclusion cannot be determined without first having knowledge of $N_{\mathrm{SFOAE}}$ in rhesus at lower levels as well. While level-dependence of $N_{\mathrm{SFOAE}}$ bears consideration when making cross-species comparisons, it does not seem likely to be a confounding factor; i.e., level-dependent effects will not account for why human delays are so much longer than other species so far examined.

There is evidence suggesting that SFOAE delays in humans become level-independent at lower stimulus intensities, with a breakpoint between $L_{\mathrm{p}}=20$ and $40 \mathrm{~dB}$ SPL [56, 2] (see Figs.5-1 and 6-8 in that latter study). Similarly for lizards: decreasing the stimulus level below $20 \mathrm{~dB}$ SPL does not appear to produce further increases in SFOAE delays [2, 5]. Such behavior appears broadly consistent with basilar membrane input/output functions being linear below 20-30 dB SPL, at least in chinchilla [42]. These observations relate to an important consideration: while the cochlea may ultimately be nonlinear even for low stimulus intensities (as evidenced by the presence of stable SOAE activity), linear properties can emerge and provide a useful basis for modeling the cochlea. Consider for example that while coherent reflection theory [63] may ultimately be linear, it can still be consistent with cochlear function when placed in a broader context that considers nonlinear aspects of amplification in order to account for SOAE generation [46]. Put another way, a cochlea behaving linearly and the existence of SOAEs are not necessarily mutually exclusive: 
the notion of 'linearity' really depends upon precisely what one is measuring/examining.

\subsection{Connections back to perception}

The discovery of eOAEs [21] was motivated by trying to understand 'ripples' apparent in the audiogram $[14,57]$. Though empirical studies examining the connections between spontaneous and evoked OAEs have been relatively limited [e.g., [10, 36]], several reports have indicated clear interrelationships that also extend back to the level of perception [e.g., [65, 29, 30, 55, 15, 27]]. Observable SOAEs correlate to minima in audiometric ("microstructure") thresholds [65, 30]. Given that SFOAE peaks correlate to those of SOAEs, SFOAE peaks (evoked via a low-level tone) might also be expected to correlate to audiometric thresholds minima [65]. Extending further, SFOAE peaks are detectable where SOAEs are not (e.g., Fig.1C). Thus, might there be additional audiometric threshold minima as well in regions where there are SFOAE peaks, but no observable SOAEs? Or rephrasing more generally, is there any sort of qualitative/quantitative relationship between SFOAE magnitude structure and audiometric threshold minima? A positive answer to such a notion, coupled with the demonstration that SFOAEs can effectively be used to estimate peripheral tuning as noted above, may indicate that low-level SFOAEs provide a more robust indicator of function rather than other commonly employed methodologies (e.g., DPOAEs, particularly those evoked at higher stimulus levels).

\subsection{Role of the middle ear}

While not explored extensively in the present study, data from one subject with abnormal middle ear function (Fig.1D) suggest that SOAEs and SFOAEs could be affected differentially. This subject had normal audiometric thresholds, but also an abnormal tympanogram indicating a hypermobile tympanic membrane. What was unique about this particular subject here was that she demonstrated robust SOAE activity, yet SFOAE magnitudes were relatively quite small (Fig.1D). In all other subjects examined here (with normal tympanograms), SOAE activity was accompanied by correspondingly strong SFOAEs near and away from SOAE peaks. Could a middle-ear 
pathology account for such? For example, SOAEs might be considered 'one way', in that they only go from cochlea to canal. Conversely, SFOAEs are 'two way' in that the stimulus goes from canal to cochlea, then the emission goes from cochlea to canal. Perhaps an additional pathway may be important for explaining the differences seen in this subject. It should prove instructive to examine whether or not a simple middle ear model could explain these differences, though such is beyond the scope of the current report. Also, the observation that SFOAE phase accumulation between SOAE peaks clusters about integer values (Fig.4B,C) suggests that middle ear filtering varies relatively slowly with frequency.

\subsection{Extending beyond the mammalian cochlea}

Previous studies have demonstrated for lizards close connections of SOAE suppression properties [25, 33] and SFOAEs phase-gradient delays [4] with auditory nerve fiber tuning curves, indicating a close relationship between emission generation mechanisms and forward transduction. Extending these observation within the context of the present study, many of the qualitative similarities observed between SOAEs and SFOAEs for humans and rhesus monkey [34] are also apparent for lizards [6,5]. For example, lizards SOAE peaks are accompanied by increased SFOAE activity, though the converse need not be true. Similarly, there is commonly integral numbers of cycles of SFOAE phase accumulation between adjacent SOAE peaks in lizards. These similarities arise despite gross morphological differences in the inner ear between humans and lizards, such as several orders of magnitude difference in the number of hair cells and the apparent lack of traveling waves along the lizard basilar membrane [39]. While such differences suggest that theoretical frameworks developed specifically within the context of the mammalian cochlea [e.g., $[55,46]]$ may not be directly applicable to lizards, a process analogous to coherent reflection has been proposed to be at work in these ears [4]. Some have hypothesized that evidence along these lines argues for a fundamental sameness between mammals and non-mammals [e.g., $[52,11]]$, although an alternative notion posits that generation mechanisms are analogous rather than homologous [13]. That is, they arose independently from systems with albeit similar constituent parts (e.g., hair cells), but 
grossly different mechanical configurations/dynamics. Additional comparative studies should help distinguish between these two hypotheses.

\section{Conclusions}

The data reported here extend and strengthen correlations between spontaneous otoacoustic emissions and those evoked using a low-level stimulus tone. Coming back to the questions initially posed:

- When SOAE peaks are present, SFOAE magnitudes show a corresponding increase in magnitude (Fig.1A,B). However, the converse need not be true: robust SFOAE activity can be observed where no SOAEs are apparent above the noise floor (Fig.1C). Thus, the correlation between the two measures is not strictly 'one-to-one'. Such an observation appears generally consistent with theoretical descriptions $[55,46,15]$

- As noted above, SFOAE activity can be comparable between subjects both with and without measurable SOAEs. Furthermore, both subject groups yield similar SFOAE phase-gradient delays (Fig.2B). Thus, SOAE peaks do not appear to strongly bias SFOAE delays for a 20 dB SPL probe level.

- Phase-gradient delays obtained with a probe level of $20 \mathrm{~dB}$ SPL are significantly longer than those obtained at $40 \mathrm{~dB}$ SPL (Fig.2A). This observation bears consideration in studies that use SFOAEs as a means to infer functional properties such as the sharpness of tuning.

Taken together, the present data provide further support for the coherent reflection model of the mammalian cochlea $[63,46]$ and indicate that agreement between model predictions and data is further improved when lower stimulus levels are used for eOAEs (Fig.3). Additionally, data from one subject with abnormal middle ear function (hypermobile tympanic membrane) indicated robust SOAEs, but minimal SFOAE activity (Fig.1D). This observation suggests that the middle ear may play a differential role between forward- and reverse-transduction relevant to OAE measurement. 


\section{Acknowledgments}

Comments from Karolina Charaziak, James Dewey, Radha Kalluri, Glenis Long, and the reviewers on the manuscript are greatly appreciated. Christopher Shera in particular provided valuable constructive/critical feedback. Financial support came from the Howard Hughes Medical Inst. (52003749) and National Science Foundation Div. of Mathematical Sciences (0602173) and the American Speech-Language-Hearing Foundation New Century Scholars Research Grant (awarded to the last author). We would like to thank Benjamin Smith for technical support. 


\section{References}

[1] Bentsen, T., Harte, J. M., and Dau, T. (2011). "Human cochlear tuning estimates from stimulus-frequency otoacoustic emissions", J. Acoust. Soc. Am. 129, 3797.

[2] Bergevin, C. (2007). "Comparative approaches to otoacoustic emissions: towards and understanding of why the ear emits sound", Ph.D. thesis, Massachusetts Institute of Technology.

[3] Bergevin, C. (2011). "Comparison of Otoacoustic Emissions Within Gecko Subfamilies: Morphological Implications for Auditory Function in Lizards", J. Assoc. Res. Otolaryngol. 12, 203-217.

[4] Bergevin, C. and Shera, C. (2010). "Coherent reflection without traveling waves: On the origin of long-latency otoacoustic emissions in lizards", J. Acoust. Soc. Am. 127, 2398.

[5] Bergevin, C., Velenovsky, D., and Bonine, K. (2011). "Coupled, active oscillators and lizard otoacoustic emissions", What Fire is in Mine Ears: Progress in Auditory Biomechanics, Melville, NY: American Institute of Physics.

[6] Bergevin, C., Velenovsky, D. S., and Bonine, K. E. (2010). “Tectorial Membrane Morphological Variation: Effects upon Stimulus Frequency Otoacoustic Emissions (Support Material)", Biophys. J. 99, 1064-1072.

[7] Bialek, W. and Wit, H. (1984). "Quantum Limits to Oscillator Stability - Theory and Experiments on Acoustic Emissions From the Human Ear", Phys. Lett. A 104, 173-178.

[8] Burns, E., Arehart, K., and Campbell, S. (1992). "Prevalence of spontaneous otoacoustic emissions in neonates", J. Acoust. Soc. Am. 91, 1571-1575.

[9] Burns, E. M. (2009). "Long-term stability of spontaneous otoacoustic emissions.”, J. Acoust. Soc. Am. 125, 3166-3176. 
[10] Burns, E. M., Strickland, E. A., Tubis, A., and Jones, K. (1984). "Interactions among spontaneous otoacoustic emissions. I. Distortion products and linked emissions.", Hear. Res. 16, 271-278.

[11] Chiappe, M. E., Kozlov, A. S., and Hudspeth, A. J. (2007). "The Structural and Functional Differentiation of Hair Cells in a Lizard's Basilar Papilla Suggests an Operational Principle of Amniote Cochleas”, J. Neurosci. 27, 11978-11985.

[12] Choi, Y., Lee, S., Parham, K., Neely, S., and Kim, D. (2008). "Stimulus-frequency otoacoustic emission: Measurements in humans and simulations with an active cochlear model”, J. Acoust. Soc. Am. 123, 2651-2669.

[13] Cooper, N. and Manley, G. (2011). "Comparative Auditory Mechanics: From Species to Species and From Base to Apex - A Moderated Discussion", What Fire is in Mine Ears: Progress in Auditory Biomechanics, Melville, NY: American Institute of Physics.

[14] Elliott, E. (1958). “A Ripple Effect in the Audiogram”, Nature 181, 1076-1076.

[15] Epp, B., Verhey, J. L., and Mauermann, M. (2010). “Modeling cochlear dynamics: Interrelation between cochlea mechanics and psychoacoustics", J. Acoust. Soc. Am. 128, 1870-1883.

[16] Goodman, S. S., Withnell, R. H., and Shera, C. A. (2003). "The origin of SFOAE microstructure in the guinea pig.", Hear. Res. 183, 7-17.

[17] Joris, P., Bergevin, C., Kalluri, R., McLaughlin, M., Michelet, P., van der Heijden, M., and Shera, C. (2011). "Frequency selectivity in Old World monkeys corroborates sharp cochlear tuning in humans", Proc. Nat. Acad. Sci. USA 108, 17516-17520.

[18] Kalluri, R. and Shera, C. A. (2007). "Comparing stimulus-frequency otoacoustic emissions measured by compression, suppression, and spectral smoothing”, J. Acoust. Soc. Am. 122, 3562. 
[19] Kalluri, R. and Shera, C. A. (2007). "Near equivalence of human click-evoked and stimulusfrequency otoacoustic emissions.”, J. Acoust. Soc. Am. 121, 2097-2110.

[20] Keefe, D. H., Ellison, J. C., Fitzpatrick, D. F., and Gorga, M. P. (2008). “Two-tone suppression of stimulus frequency otoacoustic emissions.”, J. Acoust. Soc. Am. 123, 1479-1494.

[21] Kemp, D. (1978). "Stimulated acoustic emissions from within the human auditory system.", J. Acoust. Soc. Am. 64, 1386-1391.

[22] Kemp, D. (1979). "Evidence of Mechanical Nonlinearity and Frequency Selective Wave Amplification in the Cochlea", Arch. Oto-Rhino-Laryngol. 224, 37-45.

[23] Kemp, D. T. (1980). “Towards a model for the origin of cochlear echoes.”, Hear. Res. 2, $533-548$.

[24] Koppl, C. (1995). Otoacoustic emissions as an indicator for active cochlear mechanics: a primitive property of vertebrate auditory organs (Advances in Hearing Research, World Scientific, Singapore).

[25] Koppl, C. and Manley, G. (1994). "Spontaneous otoacoustic emissions in the bobtail lizard II. Interactions with external tones", Hear. Res. 72, 159-170.

[26] Kulawiec, J. and Orlando, M. (1995). "The Contribution of Spontaneous Otoacoustic Emissions to the Click-Evoked Otoacoustic Emissions”, Ear Hear. 16, 515-520.

[27] Lee, J. and Long, G. (2011). "Stimulus characteristics which lessen the impact of threshold fine-structure on estimates of hearing status", Hear. Res. (in press) .

[28] Lineton, B. and Wildgoose, C. M. B. (2009). "Comparing two proposed measures of cochlear mechanical filter bandwidth based on stimulus frequency otoacoustic emissions", J. Acoust. Soc. Am. 125, 1558-1566. 
[29] Long, G. and Tubis, A. (1988). "Investigations Into the Nature of the Association Between Threshold Microstructure and Otoacoustic Emissions”, Hear. Res. 36, 125-138.

[30] Long, G. and Tubis, A. (1988). "Modification of Spontaneous and Evoked Otoacoustic Emissions and Associated Psychoacoustic Microstructure by Aspirin Consumption”, J. Acoust. Soc. Am. 84, 1343-1353.

[31] Long, G. R. and Talmadge, C. L. (1997). "Spontaneous otoacoustic emission frequency is modulated by heartbeat.", J. Acoust. Soc. Am. 102, 2831-2848.

[32] Lonsbury-Martin, B., Martin, G., Probst, R., and Coats, A. (1988). "Spontaneous otoacoustic emissions in a nonhuman primate. II. Cochlear anatomy.”, Hear. Res. 33, 69-93.

[33] Manley, G. (2001). "Evidence for an active process and a cochlear amplifier in nonmammals", J. Neurophysiol/ 86, 541-549.

[34] Martin, G., Lonsbury-Marin, B., Probst, R., and Coats, A. (1988). "Spontaneous otoacoustic emissions in a nonhuman primate. I. Basic features and relations to other emissions", Hear. Res. 33, 49-68.

[35] McFadden, D. and Plattsmier, H. S. (1984). "Aspirin abolishes spontaneous oto-acoustic emissions.”, J. Acoust. Soc. Am. 76, 443-448.

[36] Moulin, A., Collet, L., Veuillet, E., and Morgon, A. (1993). "Interrelations Between Transiently Evoked Otoacoustic Emissions, Spontaneous Otoacoustic Emissions and Acoustic Distortion Products in Normally Hearing Subjects”, Hear. Res. 65, 216-233.

[37] Murphy, W., Tubis, A., Talmadge, C., and Long, G. (1995). "Relaxation dynamics of spontaneous otoacoustic emissions perturbed by external tones. II. Suppression of interacting emissions", J. Acoustic. Soc. Am. 97, 3711-3720. 
[38] Neely, S., Norton, S., Gorga, M., and Jesteadt, W. (1988). "Latency of Auditory Brain-Stem Responses and Otoacoustic Emissions Using Tone-Burst Stimuli”, J. Acoust. Soc. Am. 83, $652-656$.

[39] Peake, W. T. and Ling, A. (1980). "Basilar-membrane motion in the alligator lizard: its relation to tonotopic organization and frequency selectivity.”, J. Acoust. Soc. Am. 67, 17361745.

[40] Probst, R., Coats, A., Martin, G., and Lonsbury-Martin, B. (1986). "Spontaneous, ClickEvoked, and Toneburst-Evoked Otoacoustic Emissions From Normal Ears”, Hear. Res. 21, $261-275$.

[41] Probst, R., Lonsbury-Martin, B., and Martin, G. K. (1991). “A review of otoacoustic emissions”, J. Acoust. Soc. Am. 89, 2027.

[42] Ruggero, M., Rich, N., Recio, A., Narayan, S., and Robles, L. (1997). "Basilar-membrane responses to tones at the base of the chinchilla cochlea.”, J. Acoust. Soc. Am. 101, 21512163.

[43] Schairer, K. S., Ellison, J. C., Fitzpatrick, D., and Keefe, D. H. (2006). "Use of stimulusfrequency otoacoustic emission latency and level to investigate cochlear mechanics in human ears.”, J. Acoust. Soc. Am. 120, 901-914.

[44] Shera, C. and Guinan, J. (1999). "Evoked otoacoustic emissions arise by two fundamentally different mechanisms: A taxonomy for mammalian OAEs”, J. Acoust. Soc. Am. 105, 782798.

[45] Shera, C. and Guinan, J. (2003). "Stimulus-frequency-emission group delay: A test of coherent reflection filtering and a window on cochlear tuning", J. Acoust. Soc. Am. 113, 27622772. 
[46] Shera, C. A. (2003). "Mammalian spontaneous otoacoustic emissions are amplitudestabilized cochlear standing waves.”, J. Acoust. Soc. Am. 114, 244-262.

[47] Shera, C. A., Guinan, J. J., and Oxenham, A. J. (2002). "Revised estimates of human cochlear tuning from otoacoustic and behavioral measurements.”, Proc. Nat. Acad. Sci. USA 99, 3318-3323.

[48] Shera, C. A. and Zweig, G. (1993). "Noninvasive measurement of the cochlear traveling-wave ratio.”, J. Acoust. Soc. Am. 93, 3333-3352.

[49] Siegel, J. H., Cerka, A. J., Recio-Spinoso, A., Temchin, A. N., van Dijk, P., and Ruggero, M. A. (2005). "Delays of stimulus-frequency otoacoustic emissions and cochlear vibrations contradict the theory of coherent reflection filtering.", J. Acoust. Soc. Am. 118, 2434-2443.

[50] Sisto, R. and Moleti, A. (2007). "Transient evoked otoacoustic emission latency and cochlear tuning at different stimulus levels.”, J. Acoust. Soc. Am. 122, 2183-2190.

[51] Sisto, R., Moleti, A., and Shera, C. (2007). "Cochlear reflectivity in transmission-line models and otoacoustic emission characteristic time delays.", J. Acoust. Soc. Am. 122, 3554-3561.

[52] Stewart, C. and Hudspeth, A. (2000). "Effects of salicylates and aminoglycosides on spontaneous otoacoustic emissions in the Tokay gecko”, Proc. Nat. Acad. Sci. USA 97, 454-459.

[53] Talmadge, C., Long, G., Murphy, W., and Tubis, A. (1993). "New off-line method for detecting spontaneous otoacoustic emissions in human-subjects”, Hear. Res. 71, 170-182.

[54] Talmadge, C. and Tubis, A. (1993). "On modeling the connection between spontaneous and evoked otoacoustic emissions", in Biophysics of Hair-Cell Sensory Systems, edited by H. Duifhuis, J. Horst, P. van Dijk, and S. van Netten, 25-32 (World Scientific Press).

[55] Talmadge, C., Tubis, A., Long, G., and Piskorski, P. (1998). "Modeling otoacoustic emission and hearing threshold fine structures", J. Acoust. Soc. Am. 104, 1517-1543. 
[56] Talmadge, C. L., Tubis, A., Long, G. R., and Tong, C. (2000). "Modeling the combined effects of basilar membrane nonlinearity and roughness on stimulus frequency otoacoustic emission fine structure”, J. Acoust. Soc. Am. 108, 2911.

[57] Thomas, I. (1975). "Microstructure of the pure-tone threshold", J. Acoust. Soc. Am. 57, S26.

[58] van Dijk, P., Maat, B., and de Kleine, E. (2011). "The effect of static ear canal pressure on human spontaneous otoacoustic emissions: spectral width as a measure of the intra-cochlear oscillation amplitude.”, J. Assoc. Res. Otolaryngol. 12, 13-28.

[59] van Hengel, P., Duifhuis, H., and van den Raadt, M. (1996). "Spatial periodicity in the cochlea: the result of interaction of spontaneous emissions?”, J. Acoust. Soc. Am. 99, 35663571 .

[60] Whitehead, M. (1991). "Slow variations of the amplitude and frequency of spontaneous otoacoustic emissions", Hear. Res. 53, 269-280.

[61] Whitehead, M. L., Kamal, N., Lonsbury-Martin, B. L., and Martin, G. K. (1993). "Spontaneous otoacoustic emissions in different racial groups.", Scand. Audiol. 22, 3-10.

[62] Zurek, P. (1981). "Spontaneous Narrowband Acoustic-Signals Emitted by Human Ears”, J. Acoust. Soc. Am. 69, 514-523.

[63] Zweig, G. and Shera, C. A. (1995). "The origin of periodicity in the spectrum of evoked otoacoustic emissions.”, J. Acoust. Soc. Am. 98, 2018-2047.

[64] Zwicker, E. (1990). "Otoacoustic emissions in research of inner ear signal processing", in Cochlear Mechanisms and Otoacoustic Emissions, edited by F. Grandori, G. Cianfrone, and D. Kemp, 63-76 (Karger).

[65] Zwicker, E. and Schloth, E. (1984). "Interrelation of different oto-acoustic emissions.", J. Acoust. Soc. Am. 75, 1148-1154. 